\title{
Auditory and Spatial Assessment in Inattentive Children Using Smart Devices and Gesture Interaction
}

\author{
Mauricio Loachamín-Valencia; M.-Carmen Juan \\ Instituto Universitario de Automática e \\ Informática Industrial \\ Universitat Politècnica de València \\ $\mathrm{C} /$ Camino de Vera, s/n \\ 46022-Valencia, Spain \\ \{mrenan, mcarmen\}@dsic.upv.es
}

\author{
Magdalena Méndez-López \\ Departamento de \\ Psicología y Sociología \\ Universidad de \\ Zaragoza \\ Zaragoza, Spain \\ mmendez@unizar.es
}

\author{
Elena Pérez-Hernández \\ Departamento de Psicología \\ Evolutiva y de la Educación \\ Universidad Autónoma \\ de Madrid \\ Madrid, Spain \\ elena.perezh@uam.es
}

\begin{abstract}
We present an interactive game for assessing auditory and spatial memory. We compared the performance of children with and without inattention using the game and using a variety of classical tools for assessment of auditory and spatial memory. The children with inattention showed statistically worse performance in the game. There were no statistically significant differences between the two groups for the satisfaction and interaction outcomes. Therefore, our game could be a good tool for distinguishing performances of children with and without inattention.
\end{abstract}

Keywords- game-based assessment; gesture interaction; inattention; smart devices; software for psychology

\section{INTRODUCTION}

Classical methods for testing cognitive skills are sometimes not exciting, especially for children. However, they are of interest for the psychological assessment of children's cognitive profile, especially in the field of cognitive disabilities. Beside this, one of the most frequent behaviors of children is inattention [1]. The symptoms of inattention represent a problem when children participate in different learning contexts that require substantial amounts of attention. Therefore, we need to develop assessment tools that facilitate keeping children's attention focused on the task while they are learning. In this regard, computer technology has been incorporated in psychology to improve the engagement of children during learning or assessment experiences. Computer-based, Kinect-based, and videogame tasks have shown positive results even in children with attention difficulties [2], [3]. We suggest that they could be good options for testing spatial assessment for auditory stimuli of children with symptoms of inattention. Knowing more about how inattentive children learn this type of information is of great interest. The classical psychological assessment tools have shown spatial-related deficits on a variety of specialized tests [4]. These deficits have implications for daily life and academic outcomes [4]. However, to our knowledge, no work has yet been conducted to test auditory spatial memory in children with attention difficulties using a game based on smart devices and gesture interaction. We suggest that this game might be useful in this population for several reasons: it reduces the efforts of attention required by the user to interact with the system; it allows the creation of an attractive environment; it facilitates motivation in players; and it allows the stimuli presentation and the objective registration of the performance variables to be controlled.

The goals of the study are: (1) to develop an assessment game that is able to recognize gestures and integrate gesture recognition with smart devices; (2) to obtain indicators of the participating children on traditional tools for the assessment of spatial memory and auditory memory; (3) to compare the performance obtained by using the game and the traditional tools between the two groups of the study (i.e., the inattentive group and the control group); (4) to compare the outcomes obtained on a questionnaire about satisfaction and interaction between the two groups.

Our main hypotheses are: (1) there would be statistically significant differences for the outcomes obtained in the game and the traditional tools between the children with symptoms of inattention and the children without symptoms of inattention; (2) there would not be statistically significant differences in the outcomes for satisfaction and interaction with the game between the children with symptoms of inattention and the children without symptoms of inattention.

\section{METHODOLOGY}

\section{A. General Description of the Game}

The game uses gesture interaction to recognize movements (Kinect ${ }^{\mathrm{TM}}$ ) and smart devices with auditory stimuli (Karotz rabbits) as the visual interface. The game is a serious game. The game tests the child's ability to detect and localize auditory stimuli that are emitted in different positions of a game area (see Fig. 1). The walls were covered with wrapping paper to eliminate any spatial cues. From the player's perspective, the game consisted of guessing the rabbit that emitted a sound. The rabbits were identical, so the only difference among them was their placement in the room. The advice to the player is to concentrate on the rabbits' locations and memorize them. Kinect was used to detect that the players raise their arms in front of a Karotz rabbit. This action indicates that the player has selected the Karotz rabbit that is in front of him or her (see Fig. 2).

Each Karotz rabbit emitted its assigned sound. The game included a total of 45 acoustic stimuli, which were randomly emitted in different locations to avoid repetitions or established sequences. The game is composed of five different levels based on the number of stimuli presented in each trial. Each level is related to a specific theme which includes: nature, a party, a farm, a house, and a big city. The 


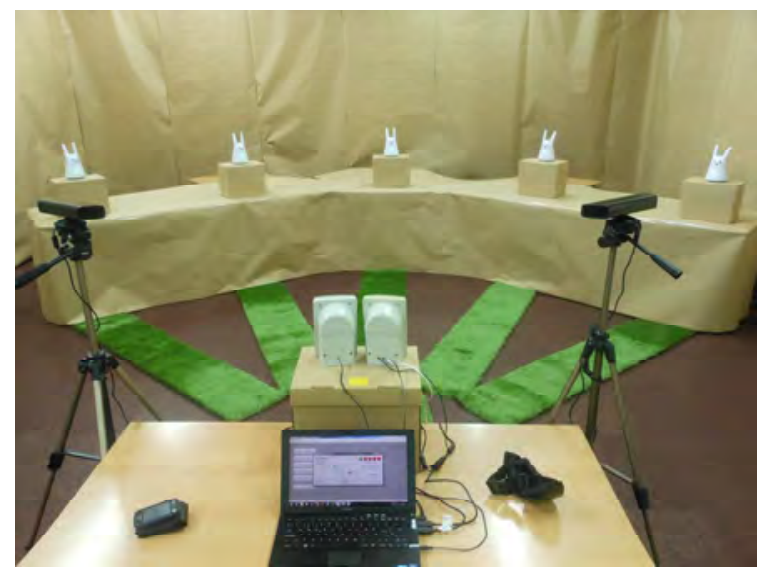

Figure 1. Game area.

acoustic stimuli were distributed as follows: Level I (1 acoustic stimulus for each trial, 3 stimuli in total); Level II (2 acoustic stimuli for each trial, 6 stimuli in total); Level III (3 acoustic stimuli for each trial, 9 stimuli in total); Level IV (4 acoustic stimuli for each trial, 12 stimuli in total); and Level $\mathrm{V}$ (5 acoustic stimuli for each trial, 15 stimuli in total).

The game had two phases: the detection phase and the location phase. In the detection phase, the children first listened to instructions through the loudspeakers. Then, they listened to the stimulus that the Karotz rabbit emitted and learned its location. The stimuli were emitted constantly. Then, the children moved to the location and raise their arms in front of the Karotz rabbit in order to select it (detection). In the location phase, the children first listened to instructions through the loudspeakers and the stimulus that had to be located. In the detection phase, the children had to remember the location from where the stimulus was emitted (sound). The stimuli were only emitted once. Then, the children had to move to the correct location and raise their arms in front of the Karotz rabbit in order to select it. Afterwards, they returned to the initial position. Their answers were recorded as successes or failures. In order to test a child's ability to recall a higher number of stimuli and locations, the number of stimuli and locations increased based on the progress at each level. The number of chances for completing a level was determined by the number of successes and/or failures. If all of the stimuli of a given trial were located correctly, then the trial was successful. However, if a stimulus of the trial was not correctly located, then the trial was a failure. If there was at least one successful trial within the three trials of a given level, the children could advance to the next level. In any case, the children have to perform the three trials. However, if they failed the three trials at any of the levels, the game ended. This was to keep the child from becoming frustrated with a higher number of incorrect responses that would impact subsequent performance on the game. Also, the game ended when the participant completed Level V.

\section{B. Hardware and Software}

We used five Karotz rabbits and two Microsoft Kinect v1 devices for our game. The Karotz rabbits are shaped like a

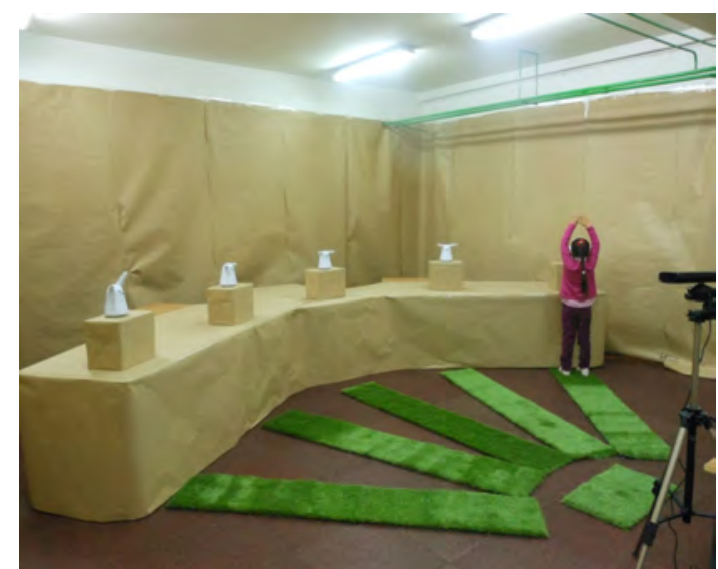

Figure 2. Child rasing her arms in front of a Karotz rabbit.

rabbit and are $30 \mathrm{~cm}$ tall (Fig. 1). They can connect to the Internet through a wireless access point. They have loudspeakers, a webcam, an LED-light (in their bellies), and they can move their ears. The Microsoft Kinect v1 devices include a RGB camera with a resolution of $640 \times 480$ pixels, an infrared camera, an infrared projector, and a multiarray microphone.

We used an HP computer with an Intel i5 processor and Windows 7 operating system. This computer had USB ports connected to a separate USB host controller. This allowed two Kinect to be used simultaneously. Additionally, this computer was used as the server. We used a wireless-G Router with WAN port for networking and accessing to Internet. This Internet access was required by the five Karotz rabbits and the computer. Two conventional loudspeakers were used to give instructions during the game (see Fig. 1).

The system that manages the procedure during the game and the graphical interface for the supervisor was developed with VisualBasic 2008 Express Edition. To program the Kinect device, we used Visual C++ 2010 Express Edition, Kinect SDK 1.8, OpenNI 2.0 SDK, and Nite. The system has three modules: one to configure and manage the Karotz rabbits, their IPs, and the IP of the sounds server; one to register the participant's information and for the evaluation process; one to manage the communication among the Karotz rabbits and the Kinect devices.

When the communication module is executed, the system receives data from the two Kinect devices, and the information of the Karotz rabbit chosen. After receiving this information, a function sends an activation message to the selected Karotz rabbit. This selected Karotz rabbit executes the function for emitting the message to the player, turning on lights, and moving its ears. The lights can be of different colors. The color green in the belly of a Karotz rabbit indicates that the system is ready to start. The color blue indicates success. The color orange indicates failures. The color white indicates that the Karotz rabbit is expecting the player's response. The color red indicates missed communication with the server.

The rest of the Karotz rabbits enter in a waiting process. Then, the selected Karotz rabbit sends a message to the system for the rest of the Karotz rabbits to end the waiting 
process and to execute the reset function to reset and start the next communication loop.

The management module of the Karotz rabbits contains a function that runs the services that were developed for each Karotz rabbit. These services allow the recognition of the IP of the selected Karotz rabbit, assign the auditory stimulus that must be emitted, and decide whether the Karotz rabbit must enter in the waiting process, the emission state, or the reset process.

The system has a graphical interface that allows the supervisor to introduce the player's information and observe the performance carried out by the player (i.e., trials, successes, and failures). The database of the system recorded successes or failures in the detection and location of stimuli.

\section{Study Sample}

A total of 34 children participated in the study. The group with symptoms of inattention (the IN Group) was comprised of 17 children (boys $=9$, girls $=8$ ). This group scored within the clinically significant range in the Attention Problems subscale of the Behavioral Assessment Scale for Children Parent Report form (BASC) [5]. The participants of the control group (the C Group) were 17 children who scored within the range of normality in the BASC (boys $=9$, girls = 8). The IN Group and the $C$ Group were matched by age and gender: 6-year-olds (one boy and four girls); 8-year-olds (three boys and one girl); 10-year-olds (five boys and three girls). The children's Intelligence Quotient (IQ) was similar for the two groups (IN Group IQ score (mean \pm standard deviation) $=103.65 \pm 11.66, \mathrm{C}$ Group IQ score (mean \pm standard deviation $)=107.47 \pm 11.82,\left(\mathrm{~F}_{1,32}=0.901, \mathrm{p}=\right.$ 0.35)). The IQ score was calculated using the RIST [6].

\section{Measures Used in the Study}

We assessed the children's memory ability during the game by considering the following variables: Percentage of Correct Trials (PCT), Percentage of Correct Stimuli (PCS), Number of Correct Levels (NCL), Time (T), Number of Errors (E) and Approximation-Errors (AE). PCT was the percentage of trials that a player could successfully complete. PCS was the percentage of stimuli that a player could successfully locate. NCL was the sum of the number of levels correctly performed. T was the total time in seconds that a player spent to complete the game. E was the sum of the number of errors that a player could commit. Finally, AE was the sum of the number of errors committed by pointing to a spatial location that was placed adjacent to the correct location.

\section{E. Assessment with Traditional Methods}

For the assessment of visuospatial memory, we selected the TOMAL [7] subtest: Memory for Location (ML). This subtest consists of a spatial recall task of one or more dots that appear within a grid printed on paper. In order to assess immediate retrieval of auditory items, we used two verbal span subtests of the TOMAL: Digits Forward (DF) and Digits Backward (DB). The DF is a number recall task that measures low-level rote recall of a sequence of numbers. The DB task (a variation of the DF task) consists of a recall of a sequence of numbers but in reverse order. For the assessment of auditory and phonological discrimination, we selected the EDAF [8] subtests: Auditory Sequential Memory (ASM), Environmental Sound Discrimination (ESD), and Auditory Figure-Ground Discrimination (AFGD). The ASM is a word repetition task. The ESD is a discrimination task about sounds of the environment that are played on a CD. Finally, the AFGD is a discrimination task in which the participant listens to two sounds simultaneously for a few seconds and must point to the two pictures that correspond to the sounds.

\section{F. Satisfaction and Interaction with the Game}

We designed a Satisfaction and Interaction Questionnaire (SIQ). The SIQ included questions about satisfaction (SQ), and questions about the interaction with the game (IQ) (Table I). The questions used a Likert scale (1-5) (1 - low and 5 - high).

\section{G. Procedure of the Study}

The parents of all the potential participants completed the Parent Report form of BASC [5]. Afterwards, the children completed the RIST [6]. Participants were tested individually in two sessions, which were conducted on a classroom. In the first session, each participant did the activities using the traditional methods. The sequence was as follows: DF, ML, DB, ASM, ESD, and AFGD. In the second session, each participant interacted with the game. Once the participant had completed this game, he/she answered the SIQ. The participants were accompanied by a supervisor. The supervisor administered the activities and questionnaires. The study was conducted in accordance with the European Directive 2001/20/EC and the Helsinki Declaration for biomedical research involving humans. The research protocol was approved by the Ethics Committee of the Universitat Politècnica de València (Spain).

\section{H. Statistical Analysis}

Levene's test was applied to check the assumption of homogeneity variance for all of the measures. The $\mathrm{E}$ and $\mathrm{AE}$ variables did not fit the normal distribution, so we conducted the Mann-Whitney $U$ test for unpaired data. For the remaining variables, a one-way ANOVA was conducted to evaluate differences between the IN Group and the C Group. Mann-Whitney $U$ tests were performed and $r$ effect sizes were calculated for the SIQ data. In all instances, significance was accepted when $\mathrm{p}<.05$.

\section{RESULTS}

\section{A. Outcomes Using the Game}

The IN Group performed worse in the game than the $\mathrm{C}$ Group (see Table II). The results of the comparisons indicated statistically significant differences for the variables related to successes (PCT, PCS, and NCL). Also, the IN Group committed a significantly higher number of errors (E and AE). There were no statistically significant differences in the time spent by each group to complete the game. 


\section{B. Outcomes Using Traditional Methods}

Table III shows the results and statistics. The performance scores on the subtest ML did not show significant differences between the two groups. Based on this subtest, both groups had similar visuo-spatial memory. Similarly, there were no statistically significant differences between the two groups in the performance scores indicating the participant's ability to discriminate sounds (ASM, ESD and AFGD). Both groups had similar auditory and phonological discriminations skills. The scores of the two verbal span subtests (DF and DB) showed that the two groups had similar ability to recall a sequence of numbers in reverse order (DB), but the IN Group did worse than the $\mathrm{C}$ Group on the forward version (DF).

\section{Satisfaction and Interaction Outcomes}

There were no statistically significant differences between the C Group and the IN Group. The children's satisfaction was favorable, as shown in Table IV. In addition, the scores related to usability were very high.

\section{DISCUSSION AND CONCLUSIONS}

To our knowledge, no study has tested this type of assessment using the technology proposed by us. Similarly, there are no published tools that focus on the assessment of auditory-spatial memory in children with symptoms of inattention.

The outcomes of children with symptoms of inattention were not as good as the outcomes obtained by children without these symptoms for the game or for the traditional method of testing the memory of verbal sounds. However, they had no difficulties for auditory discrimination of verbal and environmental sounds. We believe that our game promoted greater cognitive effort. Therefore, we suggest that the cognitive effort needed to solve this game plays a crucial role in the detection of the difficulties presented by children with symptoms of inattention. The game requires constant attention and concentration. Children with inattention have difficulty sustaining attention over time [1]. Besides these problems, there is also impaired performance on tasks of spatial memory [9].

The symptoms of inattention did not affect the time spent to complete the game. The features of our game resulted in an engaging activity. Several studies showed that inattentive children were good on computer-based tasks [2]. We suggest that our game can help to keep the participant's attention because he/she can move with some freedom in a new fun environment. This is an advantage of our game, since most children who experience this disorder have a low level of motivation to complete demanding tasks [10]. There seem to be several possible motivations for players: the physical immersion, the ability to move one's body, the ability to get feedback from the devices, and the perceptions of the game as a real experience.

The results indicate that our game was easy to use. Several authors argued that perceived ease of use is an important technical factor that affects educational effectiveness [11]. The children with symptoms of
TABLE I. SATISFACTION AND INTERACTION QUESTIONNAIRE (SIQ)

\begin{tabular}{|c|l|}
\hline \#Q & \multicolumn{1}{|c|}{ Question } \\
\hline SQ1 & How much fun did you have? \\
\hline SQ2 & Would you recommend this game to your friends? \\
\hline SQ3 & Would you like to use this game on another occasion? \\
\hline SQ4 & Score the game from 1 to 5 \\
\hline IQ1 & How easy was the game to use? \\
\hline IQ2 & Did you understand the rules of the game? \\
\hline IQ3 & Was it easy to select the sounds by raising your arms? \\
\hline IQ4 & Did you like the interaction with the rabbits? \\
\hline
\end{tabular}

TABLE II. ONE-WAY ANOVA AND MANN-WHITNEY U TEST FOR THE OUTCOMES OBTAINED USING THE GAME

\begin{tabular}{|c|c|c|c|c|c|}
\hline & C & IN & d.f. & $\mathbf{F}$ & $\mathbf{p}$ \\
\hline PCT & $84.60 \pm 4.40$ & $61.34 \pm 4.83$ & 1,32 & 12.64 & $.001^{*}$ \\
\hline PCS & $88.43 \pm 4.42$ & $66.73 \pm 4.79$ & 1,32 & 11.06 & $.002 *$ \\
\hline NCL & $3.47 \pm 0.35$ & $1.94 \pm 0.29$ & 1,32 & 11.13 & $.002 *$ \\
\hline \multirow[t]{2}{*}{$\mathbf{T}$} & $582.0 \pm 47.0$ & $540.0 \pm 53.0$ & 1,32 & 0.36 & .550 \\
\hline & $\mathrm{C}$ & IN & $U$ & $Z$ & $\mathbf{p}$ \\
\hline $\mathbf{E}$ & $2.29 \pm 0.61$ & $14.35 \pm 0.15$ & 40.50 & -3.61 & $<.001^{*}$ \\
\hline $\mathbf{A E}$ & $1.58 \pm 0.36$ & $5.35 \pm 0.14$ & 46.00 & -3.43 & $.001 *$ \\
\hline
\end{tabular}

TABLE III. ONE-WAY ANOVA FOR THE OUTCOMES OBTAINED USING THE TRADITIONAL METHODS

\begin{tabular}{|l|c|c|c|c|c|}
\hline & $\mathbf{C}$ & $\mathbf{I N}$ & d.f. & $\mathbf{F}$ & $\mathbf{p}$ \\
\hline ML & $13.06 \pm 0.89$ & $11.00 \pm 1.07$ & 1,32 & 2.17 & .150 \\
\hline DF & $8.70 \pm 0.61$ & $6.52 \pm 0.47$ & 1,32 & 7.96 & $.008^{*}$ \\
\hline DB & $11.05 \pm 0.59$ & $10.64 \pm 0.75$ & 1,32 & 0.18 & .670 \\
\hline ASM & $9.70 \pm 0.49$ & $9.41 \pm 0.49$ & 1,32 & 0.18 & .675 \\
\hline ESD & $14.17 \pm 0.23$ & $14.35 \pm 0.15$ & 1,32 & 0.41 & .523 \\
\hline AFDG & $5.29 \pm 0.19$ & $5.35 \pm 0.14$ & 1,32 & 0.06 & .806 \\
\hline
\end{tabular}

Data represent Mean \pm SEM values on the C Group and IN Group. * indicates significant differences. $\mathrm{C}=$ Control group, $\mathrm{IN}=$ Inattention group, $\mathrm{ML}=$ Memory for Location, $\mathrm{DF}=$ Digits Forward, DB = Digits Backward, ASM = Auditory Sequential Memory, ESD = Environmental Sound Discrimination, AFDG = Sound Discrimination and Pointing task.

TABLE IV. SATISFACTION AND INTERACTION OUTCOMES

\begin{tabular}{|l|c|c|c|c|c|c|}
\hline \#Q & $\mathbf{C}$ & $\mathbf{I N}$ & $\boldsymbol{U}$ & $\mathbf{Z}$ & $\mathbf{p}$ & $\mathbf{~}$ \\
\hline SQ1 & {$[5] ;[0]$} & {$[5] ;[0]$} & 161.5 & 1.049 & .601 & .180 \\
\hline SQ2 & {$[4] ;[1]$} & {$[4] ;[1]$} & 157.0 & 0.478 & .748 & .082 \\
\hline SQ3 & {$[5] ;[1]$} & {$[5] ;[1]$} & 130.0 & -0.558 & .645 & .096 \\
\hline SQ4 & {$[5] ;[1]$} & {$[5] ;[0]$} & 138.5 & -0.269 & $>.99$ & .046 \\
\hline IQ1 & {$[4] ;[1]$} & {$[4] ;[1]$} & 158.5 & 0.522 & .633 & .089 \\
\hline IQ2 & {$[5] ;[0]$} & {$[5] ;[1]$} & 179.5 & 1.634 & .178 & .280 \\
\hline IQ3 & {$[5] ;[1]$} & {$[5] ;[1]$} & 158.0 & 0.562 & .616 & .096 \\
\hline IQ4 & {$[5] ;[1]$} & {$[5] ;[0]$} & 125.0 & -0871 & .438 & .149 \\
\hline \\
Mann-Whitney U test analysis and r effect size for the Satisfaction and Interaction \\
ionnaire, and C Group and IN Group. [];[] indicate [Medians];[Interquartile range].
\end{tabular}


inattention had fun while they interacted. This is a very important result as they have difficulty maintaining their commitment to the task or remaining motivated towards achieving a goal [10]. The results indicate that the children liked interacting with their own body movements (Kinect), and they perceived the interaction with the Karotz rabbits as being easy and fun. The gesture detected by Kinect is understood as a magical way of communication with the rabbits, increasing the attractiveness of the game. These results are in line with a study showing that a Kinect-based game was interesting and an engaging activity for children [12].

Our results are also in line with a work suggesting that natural interaction with the user positively influences the perception of performance and quality of the interaction with the devices [13]. This may also be due to the physical aspect of the Karotz, the movement of their ears, the messages emitted, and the bright colors on their bellies when the child gets a response. Our informal observations confirmed that most of the children were unaware of the supervisor and that sometimes they spoke to the rabbits. The possible influence of the novelty effect on the positive perception of the system should also be taken into account [14].

We conclude that our game offers a great opportunity to assess both auditory and spatial information in children. The game is a good tool for distinguishing these difficulties in children with symptoms of inattention. Knowledge about how they learn in different smart environments provides a basis for understanding their cognitive strengths and weaknesses, besides contributing to the development of new ways of learning. Other factors that could impair performance of the group with inattention (i.e., intelligence and sound discrimination) were controlled. In addition, the memory difficulties of the IN group did not compromise their favorable perception about the game. Our game facilitates the control of the presentation of stimuli and the recording of responses. We assume that a supervisor of the experience could make some mistakes due to distraction and/or tiredness, among other causes.

Nevertheless, our specific proposal has a drawback since the Karotz rabbits are no longer being manufactured. However, other types of affordable smart devices that allow interaction are available. Therefore, our idea could serve as a basis for the development of new systems.

\section{ACKNOWLEDGMENTS}

This work was mainly funded by the Spanish Ministry of Economy and Competitiveness (MINECO) through the CHILDMNEMOS project (TIN2012-37381-C02-01) and cofinanced by the European Regional Development Fund (FEDER).

Other financial support was received from the Government of the Republic of Ecuador through the
Scholarship Program of the Secretary of Higher Education, Science, Technology and Innovation (SENESCYT), the Conselleria d'Educació, Investigació, Cultura i Esport through the grant for consolidable research groups (20172018), the Government of Aragon (Department of Industry and Innovation), and the European Social Fund for Aragon.

We would like to thank their contributions to Jimena Bonilla; the users who participated in the study; and the reviewers for their valuable comments.

\section{REFERENCES}

[1] C. Williams, B. Wright, and I. Partridge, "Attention deficit hyperactivity disorder-a review,” Br. J. Gen. Pract., vol. 49, July1999, 563-571.

[2] A. S. Drigas, R. E. Ioannidou, G. Kokkalia, and M. D. Lytras, "ICTs, mobile learning and social media to enhance learning for attention difficulties,” J. UCS, vol. 20, October 2014, pp. 1499-1510, doi:10.3217/jucs-020-10-1499

[3] S. Retalis, M. Boloudakis, G. Altanis, and N. Nikou, "Children with motor impairments play a kinect learning game: First findings from a pilot case in an authentic classroom environment,” IxD\&A, vol. 19, 2013, pp. 91-104.

[4] B. P. Rourke, "Arithmetic disabilities, specific and otherwise: a neuropsychological perspective,” J. Learn. Disabil., vol. 26, April 1993, pp. 214-226, doi:10.1177/002221949302600402

[5] C. R. Reynolds, and R. W. Kamphaus, BASC Behavior assessment system for children, Interpretative manual. Madrid, Spain: Pearson Education, 1992.

[6] C. R. Reynolds, and R. W. Kamphaus, RIST Reynolds Intellectual screening test. Interpretative manual. Madrid, Spain: TEA Ediciones, 2009.

[7] C. R. Reynolds, and E. D. Bigler, TOMAL Test of memory and learning: Examiner's manual. Madrid, Spain: TEA Ediciones, 2001.

[8] M. F. Brancal, F. Alcantud, A. M. Ferrer, and M. E. Quiroga, EDAF: Evaluación de la discriminación auditiva y fonológica (2nd ed.). Madrid, Spain: TEA Ediciones, 2009.

[9] S. Arai, Y. Okamoto, T. Fujioka, K. Inohara, M. Ishitobi, et al., "Altered frontal pole development affects self-generated spatial working memory in ADHD,” Brain Dev., vol. 38, May 2016, pp. 471-480, doi:10.1016/j.braindev.2015.11.005

[10] C. B. Holroyd, T. E. Baker, K. A. Kerns, and U. Müller, "Electrophysiological evidence of atypical motivation and reward processing in children with attention-deficit hyperactivity disorder," Neuropsychologia, vol. 46, July 2008, pp. 2234-2242, doi:10.1016/j.neuropsychologia.2008.02.011

[11] P.-C. Sun, R. J. Tsai, G. Finger, Y.-Y. Chen, and D. Yeh, "What drives a successful e-Learning? An empirical investigation of the critical factors influencing learner satisfaction," Comput. Educ., vol. 50, May 2008, pp. 1183-1202, doi: 10.1016/j.compedu.2006.11.007

[12] B. D. Homer, C. K. Kinzer, J. L. Plass, S. M. Letourneau, D. Hoffman, et al., "Moved to learn: The effects of interactivity in a Kinect-based literacy game for beginning readers," Comput. Educ., vol. 74, May 2014, pp. 37-49, doi:10.1016/j.compedu.2014.01.007

[13] M. Y. Lim, W. C. Ho, R. Aylett, and J. Dias, "Human-like memory retrieval mechanisms for social companions," Proc. Int. Conf. Autonomous Agents and Multiagent Systems (AAMAS11), vol. 3, May 2011, pp. 1117-1118.

[14] J. D. Wells, D. E. Campbell, J. S. Valacich, and M. Featherman, "The effect of perceived novelty on the adoption of information technology innovations: A risk/reward perspective,” Decision Sci., vol. 41, November 2010, pp. 813-843, doi:10.1111/j.1540-5915.2010.00292. 